\title{
Molecular classification and molecular targeted therapy of cancer
}

\author{
Miao Xu (ه), Jianyong Shao, Yixin Zeng \\ Department of Research and Molecular Diagnostics, Sun Yat-sen University Cancer Center, the State Key Laboratory of Oncology in South \\ China, Guangzhou 510060, China \\ (C) Higher Education Press and Springer-Verlag Berlin Heidelberg 2013
}

Various molecular analytical techniques have provided more and more information which is necessary for the accurate classification of diseases. Such information has enabled researchers to shift from morphologybased classification to molecular characteristics-based classification, also known as molecular classification. Molecular classifications of diseases can be carried out at the DNA, RNA, and protein levels. At the DNA level, for example, molecular classification can be done based on genetic mutation, polymorphisms, cytogenetic changes in genomes or DNA methylation differences. Classification based on different mRNA expression profiles is currently a key research approach. Since its introduction in the 1990s, biochip technology, as a highthroughput large-scale analytic technology, has been increasingly applied in research on functional genomics, disease genomics, and pharmacogenomics. Rapid advances in molecular classification have shed light on the establishment of guidelines for the molecular classification of tumors and personalized diagnosis and treatment.

Tumors are a heterogeneous group of systemic diseases that develop in multiple steps/phases. Although loss of genomic stability and evading immune destruction are two milestone events that may be observed throughout the occurrence and development of tumors, the pathogenesis of tumors differs widely among different cancers and individuals. Dramatic advances in basic research over the last 30 years have enabled practitioners to understand the pathogenesis of malignancies at the molecular level and provided solid bases for the prevention and treatment of tumors. The prognoses of most malignancies, advanced malignant tumors in particular, have unfortunately not been fundamentally improved.

Histopathology has long been the "gold standard" of

Correspondence: xumiao@sysucc.org.cn tumor diagnosis and the basis for clinical treatment; however, for tumors of the same phases and stages, treatment responses and prognoses may differ vastly among patients even after application of the same therapeutic strategy. In fact, malignant tumors are highly heterogeneous at the molecular level. Tumors with the same morphology may contain varied changes in molecular genetics, resulting in diversity in treatment responses and prognoses. Individualized treatment based on molecular differences is thus a new direction for tumor treatment, and molecular classification is the basis of individualized therapy.

One of the challenges in the new century is to determine specific targeted therapies aimed at tumor types with the same pathological origin so as to maximize the efficacy and minimize the toxicity of therapy. Accurate classification of tumors will obviously be vital toward achieving this endeavor.

In our laboratory, we employed high-throughput molecular techniques, such as gene chip and tissue microarray to detect 18 molecular markers by immunohistochemistry and determined that the combined expression levels of seven proteins, including latency membrane protein 1 (LMP1), CD147, caveolin-1, phospho-P70S6 kinase, survivin, matrix metalloproteinase 11 (MMP11), and secreted protein acidic and rich in cysteine (SPARC), in tumors, together with patient sex, can distinguish patients with nasopharyngeal carcinoma (NPC) into low- and high-risk groups for prediction of NPC survival [1]. For these two groups of patients, the division indicates the clinical application of individualized therapy so as to prevent over-treatment of the low-risk group and ensure appropriate treatment of the high-risk group. We also detected cancer stem cells in NPC and proposed that genomic instability and DNA damage are among the main causes of cancer stem cell formation [2,3]. In fact, the expressions of many important genes, including p63, RASSF1A, VEGF, CCND1, and Hsp70, significantly differ in NPC tissue 
obtained from different patients, which we believe will affect the biological behaviors of tumors [4-8]. We further found that immune cells remarkably differ among NPC patients and that the expressions of various immune-related genes in tumor tissue are correlated with disease prognosis [9-11]. This finding is particularly important for us to understand tumor heterogeneity and carry out molecular classification and individualized treatment.

By assessing different expression levels of molecular markers and genetic variations, we may predict the effects of these factors on tumor progression and facilitate early diagnosis. Such knowledge can remedy the shortcomings of early diagnosis, conventional pathological classification, and clinical staging, improve the clinical treatment and prognosis of tumors, and thus pave the way for molecular classification and individualized treatment. Given our increased understanding of the molecular mechanisms of tumors, molecular targeted therapy (MTT), a novel approach for tumor treatment, has emerged. MTT can effectively intervene signaling pathways regulated by signature molecules and closely related to tumorigenesis and thus suppress the growth, progression, and metastasis of tumors by determining specific molecules that are over-expressed in tumor cells as targets and selecting specific blockers for these molecules. MTT findings better reflect our understanding of tumors: tumors are a systemic disease. Therefore, treatment of tumors focusing on molecular targets that are specifically or highly expressed in tumors can avoid damage to normal cells, particularly immune cells. In other words, when tumor cells are killed, the immune system, which may already be partially damaged, must be carefully protected. Some chemotherapeutic drugs can promote genomic instability and thus induce stem-like cancer cells during tumor cell attack [2].

Diagnosis and classification of tumors using molecular markers can distinguish among the different types of tumors in a more accurate manner based on gene structure and expression and thus better predict the response of a specific tumor to different treatments. For example, the development and application of molecular targeted drugs, including Bcr-Abl tyrosine kinase inhibitor imatinib, epidermal growth factor receptor (EGFR) tyrosine kinase inhibitor gefitinib, and anti-VEGF monoclonal antibody bevacizumab, are based on the molecular classifications of the corresponding tumors. Molecular classification allows more accurate tumor diagnosis and more individualized treatment; thus, it improves treatment efficacy, avoids improper application of various therapies, alleviates patient pain, and saves resources.

Research and development in molecular classification marks the emerging era of "molecular medicine" and represents a new revolution in disease management. For instance, MTT for breast cancer is mainly based on the expressions of estrogen receptor (ER), progesterone receptor (PR), and human epidermal growth factor receptor 2 (Her-2) in tumor tissue: (a) MTT targeting Her2: Trastuzumab is a recombinant DNA-derived humanized monoclonal antibody that selectively targets the extracellular domain of Her-2. It can be used for the treatment of advanced breast cancer with Her-2 overexpression. (b) MTT targeting VEGF: Bevacizumab is a recombinant humanized monoclonal antibody directed against VEGF-A subtype. It is effective for metastatic breast cancer that has received multiple cycles of treatment. (c) MTT targeting both EGFR and tyrosine kinase: Gefitinib is a potent tyrosine kinase inhibitor. It can suppress the Her-2 signaling pathway by inhibiting the tyrosine kinase domain of EGFR. The combination of trastuzumab and gefitinib can exert synergistic effects when treating Her-2-positive breast cancer. (d) Other MTT approaches: LY900003 or Affinitak ${ }^{\mathrm{TM}}$, an antisense oligonucleotide targeting the type I regulatory subunit alpha of protein kinase, can be used for treating metastatic breast cancer that is unresponsive to previous chemotherapy. In recent years, studies on the combined application of targeted drugs, antiangiogenesis drugs and conventional chemotherapeutic drugs for the treatment of cancer have became hot topics in both basic and clinical research, and many scientific breakthroughs in this area have been made.

In summary, molecular classification represents a new direction for disease diagnosis, and individualized treatment should be actively pursued in the clinical setting. With rapid advances in research on the pathogenesis of tumors, molecular targeted drugbased tailored treatments will play an increasingly important role in tumor management. The large number of tumor cases in China has urged Chinese scientists to carry out carefully designed studies on molecular classification. Under the joint efforts of clinicians and scientists, significant achievements in the research and application of molecular classification and individualized therapy of cancer can be expected, which will ultimately improve the quality of life of patients and prolong their survival.

\section{References}

1. Wang HY, Sun BY, Zhu ZH, Chang ET, To KF, Hwang JS, Jiang H, Kam MK, Chen G, Cheah SL, Lee M, Liu ZW, Chen J, Zhang JX, Zhang HZ, He JH, Chen FL, Zhu XD, Huang MY, Liao DZ, Fu J, Shao Q, Cai MB, Du ZM, Yan LX, Hu CF, Ng HK, Wee JT, Qian CN, Liu Q, Ernberg I, Ye W, Adami HO, Chan AT, Zeng YX, Shao JY. Eight-signature classifier for prediction of nasopharyngeal [corrected] carcinoma survival. J Clin Oncol 2011; 29(34): 45164525 
2. Liang Y, Zhong Z, Huang Y, Deng W, Cao J, Tsao G, Liu Q, Pei D, Kang T, Zeng YX. Stem-like cancer cells are inducible by increasing genomic instability in cancer cells. J Biol Chem 2010; 285(7): 4931-4940

3. Liang Y, Zhang H, Feng QS, Cai MB, Deng W, Qin D, Yun JP, Tsao GS, Kang T, Esteban MA, Pei D, Zeng YX. The propensity for tumorigenesis in human induced pluripotent stem cells is related with genomic instability. Chin J Cancer 2013; 32(4): 205-212

4. Guo C, Pan ZG, Li DJ, Yun JP, Zheng MZ, Hu ZY, Cheng LZ, Zeng $\mathrm{YX}$. The expression of $\mathrm{p} 63$ is associated with the differential stage in nasopharyngeal carcinoma and EBV infection. J Transl Med 2006; 4 (1): $23-30$

5. Pan ZG, Kashuba VI, Liu XQ, Shao JY, Zhang RH, Jiang JH, Guo C, Zabarovsky E, Ernberg I, Zeng YX. High frequency somatic mutations in RASSF1A in nasopharyngeal carcinoma. Cancer Biol Ther 2005; 4(10): 1116-1122

6. Liu X, Lv XB, Wang XP, Sang Y, Xu S, Hu K, Wu M, Liang Y, Liu P, Tang J, Lu WH, Feng QS, Chen LZ, Qian CN, Bei JX, Kang T, Zeng YX. MiR-138 suppressed nasopharyngeal carcinoma growth and tumorigenesis by targeting the CCND1 oncogene. Cell Cycle 2012; 11(13): 2495-2506
7. Li YH, Hu CF, Shao Q, Huang MY, Hou JH, Xie D, Zeng YX, Shao JY. Elevated expressions of survivin and VEGF protein are strong independent predictors of survival in advanced nasopharyngeal carcinoma. J Transl Med 2008; 6(1): 1-11

8. Cai MB, Wang XP, Zhang JX, Han HQ, Liu CC, Bei JX, Peng RJ, Liang Y, Feng QS, Wang HY, Chen LZ, Fu S, Kang T, Shao JY, Zeng YX. Expression of heat shock protein 70 in nasopharyngeal carcinomas: different expression patterns correlate with distinct clinical prognosis. J Transl Med 2012; 10(1): 96-107

9. Cai MB, Han HQ, Bei JX, Liu CC, Lei JJ, Cui Q, Feng QS, Wang HY, Zhang JX, Liang Y, Chen LZ, Kang TB, Shao JY, Zeng YX. Expression of human leukocyte antigen $\mathrm{G}$ is associated with prognosis in nasopharyngeal carcinoma. Int J Biol Sci 2012; 8(6): 891-900

10. Li J, Qian CN, Zeng YX. Regulatory T cells and EBV associated malignancies. Int Immunopharmacol 2009; 9(5): 590-592

11. Li J, Zhang XS, Xie D, Deng HX, Gao YF, Chen QY, Huang WL, Masucci MG, Zeng YX. Expression of immune-related molecules in primary EBV-positive Chinese nasopharyngeal carcinoma: associated with latent membrane protein 1 (LMP1) expression. Cancer Biol Ther 2007; 6(12): 1997-2004 